\title{
Über Pyrokondensationen in der aromatischen Reihe
}

(Zweite Mitteilung)

Von

Hans Meyer und Alice Hofmann

Aus dem Chemischen Laboratorium der k. k. Deutschen Universität Prag (Vorgelegt in der Sitzung am 22. Februar 1917)

In Fortsetzung unserer Versuche über die primären Zerfallsprodukte der aromatischen Verbindungen durch Überhitzung ${ }^{1}$ haben wir zunächst das

Verhalten der aromatischen Halogenverbindungen studiert.

Es wurden, soweit dies unter den gegenwärtigen Verhältnissen möglich war, charakteristische Vertreter von Ortho-, Meta- und Paraverbindungen aller vier Halogene untersucht.

In allen Fällen wird sowohl Halogen als auch Wasserstoff abgespalten; von den Halogenen, wie zu erwarten war, Jod am leichtesten, Fluor am schwersten. Jod wird zum größten Teile, Brom und Chlor spurenweise in elementarer Form erhalten, sonst als Halogenwasserstoff.

An Stelle des Halogens tritt Wasserstoff in den aromatischen Kern: Verkettung der Reste an der Stelle, wo der Substituent gewesen war, also z. B. nach dem Schema:

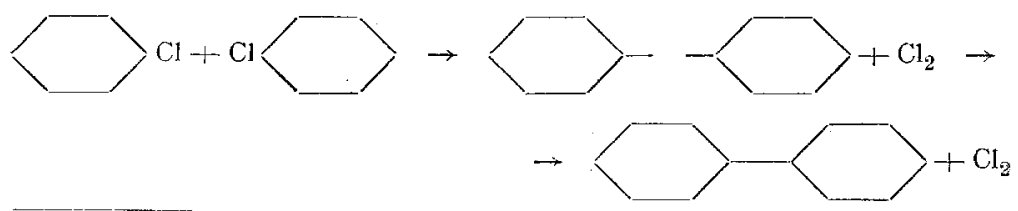

I Erste Mitteilung: Monatsh., 37, 681 (1916). 
wurde nie mit Sicherheit beobachtet: wo, wie z. B. beim Jodbenzol, kleine Mengen Diphenyl sich fanden, kann man auch die sekundäre Bildung des $Z_{w}$ eikernsystems aus dem reichlich entstandenen Benzol annehmen.

Die Halogenabspaltung tritt, mit Ausnahme der Jodverbindungen, bei den Meta- und Paraderivaten gegen die Wasserstoffabspaltung und Verkettung sehr zurück.

Von Orthoverbindungen haben wir vorläufig nur das Bromtoluol untersuchen können, das außerordentlich leicht und glatt nach der Gleichung:

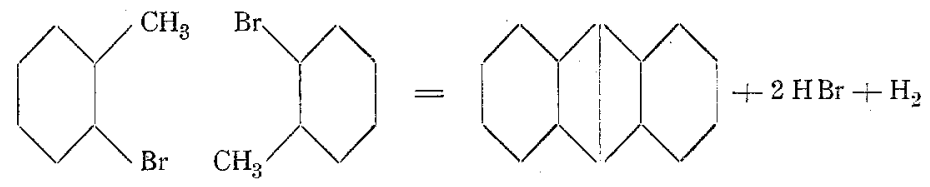

Anthracen liefert. Es dürfte wohl keinem Zweifel unterliegen, daß sich Orthochlor- und Orthojodtoluol ebenso verhalten werden. Interessanter dürfte das Resultat beim Orthofluortoluol werden, dessen Darstellung wir in Angriff genommen haben.

Bei den Monohalogenbenzolen tritt als Hauptreaktion die Bildung von parasubstituierten Diphenylen ein. Als Nebenprodukt wurde gelegentlich etwas Orthoderivat erhalten. Ist die Parastellung auch durch Halogen besetzt, so tritt Orthokondensation ein (1.1.3.3-Tetrachlordiphenyl aus $p$-Dichlorbenzol) oder das eine Halogenatom wird abgespalten (Paradibrombenzol) und man erhält aus dem ebenfalls noch nachweisbaren Monosubstitutionsprodukt die auch aus diesem direkt entstehenden Derivate.

Sehr bemerkenswert ist, daß namentlich Chlor und Brom den Benzolkern widerstandsfähiger machen: man muß, um Reaktion zu erzwingen, viel länger und auf höhere Temperatur erhitzen wie beim Benzol selbst. Fluorbenzol ähnelt dem nichtsubstituierten Stammkörper in seinem Verhalten. Beim Jodbenzol trübt die leichte Abspaltbarkeit des Halogens das Bild.

Quecksilberdampf beschleunigt die Reaktion (Brombenzol). 
Von den Toluolderivaten ist das Orthobromtoluol schon erwähnt worden. Von Metaverbindungen konnte bisher nur das $m$-Fluortoluol untersucht werden. Es liefert fast ausschließlich $m$-Fluordibenzyl. $p$-Fluortoluol dagegen läßt fast nur $p$-Fluorstilben entstehen, das gut krystallisiert. Die geringen flüssigen Mutterlaugen enthalten wahrscheinlich, neben weiteren Stilbenmengen, etwas $p$-Fluordibenzyl. Es konnte indes kein reines (festes) Produkt daraus isoliert werden. Analoges gilt vom $p$-Jodtoluol.

Beim $p$-Chlortoluol überwiegt dagegen die Beständigkeit des $p$-Dichlordibenzyls; das zugehörige Stilben konnte nur dann in greifbarer Menge erhalten werden, wenn andauernd und hoch erhitzt worden war.

Zur Anthracenbildung haben die untersuchten Halogentoluole der Meta- und Parareihe durchaus keine Neigung.

Im einzelnen ergaben die Versuche das Folgende.

\section{Chlorbenzol.}

Nach zwölfstündigem Erhitzen auf helle Rotglut war ungefähr die Hälfte der Substanz in Reaktion getreten.

Beim Fraktionieren wurde nach der Entfernung des unveränderten Chlorbenzols eine geringe Menge bei 250 bis $295^{\circ}$, dann etwas größere Mengen von 295 bis $300^{\circ}$ und die Hauptmenge bei 300 bis $305^{\circ}$ aufgefangen.

Der über $305^{\circ}$ übergehende Anteil (Siedepunkt bis $320^{\circ}$ ) erstarte nach dem Verreiben mit Äther. Die Krystallmasse wurde abgepreßt und schmolz ohne weitere Reinigung bei 139 bis $142^{\circ}$. Nach dem Umkrystallisieren aus Methylalkohol blieb der Schmelzpunkt bei 144 bis $145^{\circ}$ konstant.

Die Substanz ist mit $p$-Dichlordiphenyl identisch, für das Schmelzpunkt $148^{\circ}$. und Siedepunkt $315^{\circ}$ angegeben werden. Die Chlorbestimmung lieferte den erwarteten Wert:

$0 \cdot 203 \mathrm{~g}$ gaben $0.262 \mathrm{~g}$ Chlorsilber.

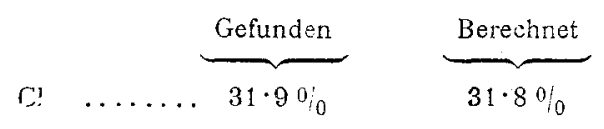


$\mathrm{Da}$ aus den unter $305^{\circ}$ übergegangenen Fraktionen in keinerlei Weise feste Anteile abtrennbar waren, wurden die einzelnen Fraktionen, alle mit gleichem Erfolg, oxydiert.

Das Ö1 wurde in Eisessig gelöst und mit der fünffachen Menge Chromtrioxyd versetzt. Die anfangs heftige Reaktion wurde durch Kühlen in Schranken gehalten, schließlich noch eine Stunde am Rückflußkühler zum Sieden erhitzt. Nach dem Erkalten wurde in Wasser gegossen, mit Lauge übersättigt und durch Ausschütteln mit Äther unangegriffene Anteile entfernt. Durch Ansäuern ließ sich aus der alkalischen Lösung eine reichliche Menge eines krystallinischen Niederschlages gewinnen, der abgesaugt, getrocknet und sublimiert wurde. Schmelzpunkt des Sublimats im geschlossenen Röhrchen 235 bis $236^{\circ}$, schöne glänzende Blättchen; Mischschmelzpunkt mit $p$-Chlorbenzoesäure unverändert.

Das Filtrat von der $p$-Chlorbenzoesäure wurde mil Äther erschöpft. Nach dem Waschen des Extraktionsmittels wurde destilliert und nach der Entfernung von Äther und des größten Teiles der mitextrahierten Essigsäure ein schwach grünlicher Rückstand erhalten, der auf Ton abgepreßt und hierauf sublimiert wurde. Die so erhaltenen schönen; glänzenden, farblosen Blättchen schmolzen bei 131 bis $135^{\circ}$. Sie wurden aus Wasser umkrystallisiert und nochmals sublimiert. Der nunmehr (im beiderseits geschlossenen Röhrchen) erhaltene Schmelzpunkt 137 bis $138^{\circ}$ blieb beim weiteren Umkrystallisieren konstant. Die Substanz ist nach Schmelzpunkt und Mischungsschmelzpunkt als Orthochlorbenzoesäure anzusprechen.

Das Filtrat vom Umkrystallisieren der Orthochlorbenzoesäure wurde auf Benzoesäuregehalt untersucht, doch konnte keine aufgefunden werden.

Ebensowenig ließ sich im Vorlauf des Rohöls Benzol oder in den höheren Fraktionen unsubstituiertes Diphenyl nachweisen.

Während des Glühens war Chlorwasserstoff- und (in geringer Menge) Chlorabspaltung festzustellen.

Die Reaktionsprodukte sind also: $p$-Dichlordiphenyl neben Monochlordiphenylen; eine Chlorbestimmung des flüssigen, von 300 bis $305^{\circ}$ übergehenden Hauptanteiles ergab einen Wert, 
der nur einige Prozente unter dem für Dichlordiphenyl berechneten lag. Es werden also noch reichliche Mengen an $p$ - (neben vielleicht $o$-) Dichlordiphenyl und Monochlordiphenyle in diesem untrennbaren Gemisch vorliegen.

\section{p-Dichlorbenzol.}

Die Reaktion erfordert sehr helle Rotglut und vielstündiges Erhitzen.

Zur Aufarbeitung des Reaktionsproduktes wurde der geschmolzene Kolbeninhalt mit Wasserdampf behandelt und dadurch kleine Mengen von Monochlorbenzol und unverändertes Dichlorbenzol abgetrennt. Die Destillation muß sehr lange fortgesetzt werden, wenn sie vollständig sein soll. Der nichtflüchtige, harzige Rückstand wurde mit Eisessig ausgekocht. Es ging fast alles in Lösung. Nach dem Abdestillieren der Hauptmenge des Lösungsmittels wurde stark abgekühlt und dadurch eine reichliche Menge gelblicher Krystalle abgetrennt, deren Schmelzpunkt durch Umkrystallisieren aus Methylalkohol und hierauf aus Petrolpentan, unter Zusatz von Tierkohle, bei 84 bis $85^{\circ}$ unverändert blieb.

Die Analyse ${ }^{1}$ erwies das Volliegen von Tetrachlordiphenyl:

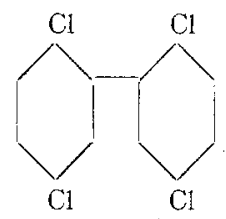

4. $297 \mathrm{mg}$ gaben $7 \cdot 87 \mathrm{mg}$ Chlorsilber.

$$
\text { Cl....... } \quad \underbrace{\text { Berechnet }}_{48.3 \%} \quad \underbrace{\text { Gefunden }}_{45 \cdot 3 \%}
$$

Andere Reaktionsprodukte konnten nicht aufgefunden werden.

\section{Brombenzol.}

Neben unverändertem Brombenzol wurden zwei Fraktionen von 270 bis $300^{\circ}$ und von 300 bis $330^{\circ}$ aufgefangen.

1 Die Substanz enthielt noch eine Spur Tierkohle. 
Da es auf keine Weise gelang, aus den Ölen etwas Festes abzuscheiden, wurden sie mit der fünffachen Menge Chromtrioxyd oxydiert. Die Reaktion war nicht sehr lebhaft. Sie lieferte eine in Wasser schwer lösliche Säure, die nach dem Umkrystallisieren und Sublimieren bei $250^{\circ} \mathrm{schmolz}$ und sich ais $p$-Brombenzoesäure erwies.

$\mathrm{Da}$ - ähnlich wie beim Chlorbenzol - die Reaktion nur langsam vor sich ging, so daß die Ausbeuten an umgewandelter Substanz recht kleine waren, wurde mit Erfolg versucht, die Zersetzung des Brombenzols durch Katalysatorwirkung $z u$ beschleunigen.

$\mathrm{Zu}$ diesem Zweck wurde das Erhitzen bei Gegenwart einiger Tropfen Quecksilber vorgenommen, dessen Dampf mit auf die Reaktionstemperatur gebracht wurde.

Die Wirkung des Quecksilbers zeigte sich einerseits in einer wesentlich vergrößerten Bromabspaltung, andrerseits aber auch darin, daß die Menge der kondensierten Substanz sehr bedeutend erhöht wurde, so daß es gelang, aus ihr einen einheitlichen Körper abzutrennen.

Aus dem Vorlauf konnte in üblicher Weise Benzol erhalten und rein dargestellt werden. Nach dem Entfernen des unveränderten Brombenzols destillierten flüssige Anteile, mit ihnen etwas Quecksilberbromid. Bis $320^{\circ}$ war, mit Ausnahme von nicht unzersetzt destillierbarem Harz, das Meiste übergegangen. Das Öl wurde mit Pentan vermischt, filtriert und auf $-80^{\circ}$ andauernd abgekühlt. Es trat reichliche Krystallisation ein. Die aus Alkohol umkrystallisierte Ausscheidung schmolz bei 87 bis $88^{\circ}$ und erwies sich als $p$-Bromdiphenyl.

\section{p-Dibrombenzol.}

Beim ersten Versuch wurde ganz ebenso vorgegangen, wie für die Aufarbeitung des Produktes aus $p$-Dichlorbenzol beschrieben wurde. Der Rückstand nach dem Abdestillieren des Eisessigs wurde nach einigem Stehen krystallinisch. Nach dem Abpressen auf Ton wurde die Substanz, die in Methylalkohol außerordentlich schwer, in Eisessig nicht leicht löslich ist, aus letzterem umkrystallisiert. Die farblosen Krystalle 
schmolzen bei 210 bis $211^{\circ}$. Die Substanz war sehr bromreich. Zur vollständigen Reinigung war ihre Menge ungenügend. Wahrscheinlich liegt Tetrabromdiphenyl

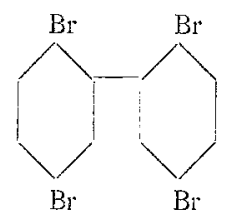

vor. Bei einem zweiten Versuche, bei dem längere Zeit erhitzt worden war, erwies sich das Reaktionsprodukt als nur mehr teilweise fest. Es wurde abgesaugt.

Der feste Anteil wurde mit Wasserdampf von der Hauptmenge des unveränderten Dibrombenzols befreit und der harzige Rückstand mit Alkohol ausgekocht. Beim Erkalten schied sich ein gelbliches Öl ab, das in der Kälte mit Methylalkohol verrieben wurde. Dadurch konnte noch eine reichliche Menge Dibrombenzol entfernt werden. Der. Rückstand krystallisierte nach längerem Stehen. Er wurde auf Ton abgepreßt und lieferte den später $z u$ besprechenden Körper vom Schmelzpunkte $117^{\circ}$.

Die äthylalkoholische Lösung enthielt anscheinend nur Dibrombenzol. Der Rückstand von der Alkoholextraktion wurde mit Eisessig ausgekocht. Nach dem Abdestillieren des Lösungsmittels wurde aber nicht, wie wir erwartet haben, der bei 210 bis $211^{\circ}$ schmelzende Körper, sondern ein Produkt erhalten, das nach wiederholtem Umkrystallisieren aus viel Alkohol konstant bei 162 bis $163^{\circ}$ schmolz und der Analyse und seinen Eigenschaften nach als $p p$-Dibromdiphenyl

anzusprechen ist.

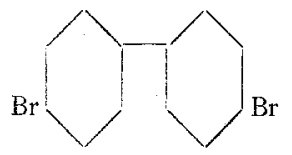

Der flüssige Anteil des Rohproduktes wurde fraktioniert. Ein Teil ging beim Siedepunkt des Brombenzols (sehr wenig), die Hauptmenge beim Siedepunkt des Dibrombenzols über. Das höher siedende wurde mit Methylalkohol gekocht 
und dadurch noch weitere Mengen Dibrombenzol entfernt. Der Rückstand erstarrte nach längerem Stehen in der Kälte. Aus viel siedendem Alkohol werden hübsche Nadeln erhalten, die bei $117^{\circ}$ schmeizen. Die Substanz sublimiert sehr schön. Die Analyse ergibt nahezu den für ein Dibromdiphenyl berechneten Bromgehalt:

$5 \cdot 613 \mathrm{mg}$ lieferten $7 \cdot 074 \mathrm{mg}$ Bromsilber.

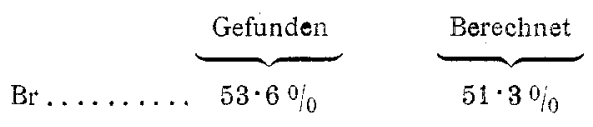

Wahrscheinlich liegt eines der möglichen Orthoderivate vor.
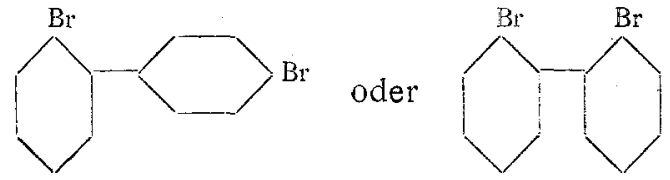

\section{Jodbenzol.}

Schon bald nach Beginn des Erhitzens macht sich die Abspaltung von Jod durch das Auftreten violetter Dämpfe bemerkbar. Nach einigen Stunden wurde unterbrochen, als das charakteristische Prasseln im Kolben die Bildung niedrig siedender Anteile in der Flüssigkeit anzeigte.

An den kupfernen Zuleitungsröhren waren graue krystalline Krusten von Kupferjodür abgeschieden. Beim Fraktionieren des Reaktionsproduktes wurde ein reichlicher Vorlauf, der unter $100^{\circ}$ siedete, abgetrennt. Schütteln mit Bisulfit entfernte kleine Jodmengen. Beim nochmaligen Fraktionieren dieses Anteiles ging fast alles beim Siedepunkt des Benzols über. Durch Ausfrieren wurde der Kohlenwasserstoff ganz rein abgeschieden und durch Verwandeln in Nitrobenzol etc. identifiziert.

Vor dem weiteren Fraktionieren wurde energisch mit Bisulfitlösung geschüttelt und getrocknet. Nach dem Abtrennen des unveränderten Jodbenzols wurden zwei Fraktionen von 250 bis $300^{\circ}$ und von 300 bis $360^{\circ}$ aufgefangen. 
Es gelang auf keinerlei Weise, aus einer der beiden Fraktionen, die beide stark jodhaltige, farblose, lichtbrechende Öle bildeten, etwas Festes abzutrennen.

Daß indessen hier ausschließlich ein Gemisch von Joddiphenylen vorliegt, ließ sich schon aus dem Siedepunkt, vor allem aber durch Reduktion des Gemisches zur Stammsubstanz, erkennen.

Jede der Fraktionen wurde für sich in heißem Alkohol gelöst und mit überschüssigem Natriumamalgam 8 Stunden zum schwachen Sieden erhitzt.

Dann wurde die alkoholische Lösung mit Salzsäure abgesättigt und in Wasser eingegossen. Es trat sofort krystalline Fällung eines reichlichen Niederschlages auf, der in Chloroform aufgenommen wurde. Der Rückstand des Lösungsmittels einmal aus wenig Alkohol umkrystallisiert, erwies sich als reines Diphenyl vom Schmelzpunkt 67 bis $68^{\circ}$ (Mischungsschmelzpunkt etc.). Außer Diphenyl waren keine anderen Substanzen zugegen.

\section{Fluorbenzol.}

Nach dem Abdestillieren des unveränderten Anteiles stieg das Thermometer rasch auf über $200^{\circ}$. Die Fraktion 200 bis $300^{\circ}$ wurde beim starken Ablühlen fast vollständig fest. Höher siedende Anteile waren nicht vorhanden.

Das abgesaugte flüssige Produkt wurde nochmals fraktioniert.

Es gingen wieder kleine Mengen Fluorbenzol über und dann eine Hauptfraktion von 230 bis $260^{\circ}$, die im Kohlensäure-Äthergemisch wieder reichliche Mengen Krystalle abschied. Die Krystallfraktionen besaßen nach dem Abpressen beide den Schmelzpunkt 94 bis $95^{\circ}$. Sie wurden vereinigt und aus Aikohol umkrystallisiert. Der Schmelzpunkt änderte sich nicht. Schöne, farblose Blättchen. Die Analyse führte zur Formel $\mathrm{C}_{12} \mathrm{H}_{8} \mathrm{Fl}_{2}$.

Die Substanz erwies sich bei näherer Untersuchung als identisch mit dem schon bekannten, als wirksamer Bestandteil des Keuchhustenmittels Antitussin dienenden $p p$-Difluor- 
diphenyl. Der erste Darsteller ${ }^{1}$ der Substanz hat den Schmelzpunkt 88 bis $89^{\circ}$ gefunden.

4. $464 \mathrm{mg}$ gaben $1.71 \mathrm{mg}$ Wasser und $12 \cdot 46 \mathrm{mg}$ Kohiendioxyd.

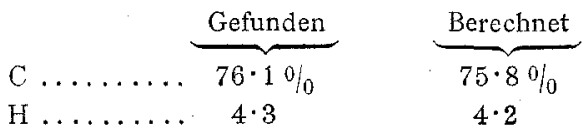

Beim Glühen des Fluorbenzols ließ sich die Abspaltung von etwas Fluorwasserstoff aus dem Irisierendwerden der Kolbenwände und dem Abblättern feiner Glasflitterchen erkennen.

\section{Orthobromtoluol.}

Beim Erhitzen trat sehr bald massenhafte Entwicklung von Bromwasserstoff auf.

Das Reaktionsprodukt lieferte nach dem Abtrennen des aus unverändertem Bromtoluol bestehenden Vorlaufes ein zwischen 260 bis $360^{\circ}$ übergehendes Destillat, das schon im Kühlrohre größtenteils erstarrte. Nach dem Absaugen einmal unter Tierkohlezusatz umkrystallisiert, war die Substanz farblos und schmolz bei $206^{\circ}$, war aber, wie die Analyse lehrte, noch bromhaltig. Erst wiederholtes Umkrystallisieren aus Alkohol, wobei in die Mutterlauge ein amorpher, an der Luft und im Licht sich rosa färbender, leicht löslicher Begleiter ging, führte zu einer reinen, bromfreien Substanz, die nach dem Schmelzpunkt $213^{\circ}$, der Überführbarkeit in Anthrachinon, in das charakteristische Pikrat und das schöne, bei 221 bis $222^{\circ}$ schmelzende 9.10-Dichlorderivat als Anthracen erkannt wurde, dessen Entstehen nach dem Schema:

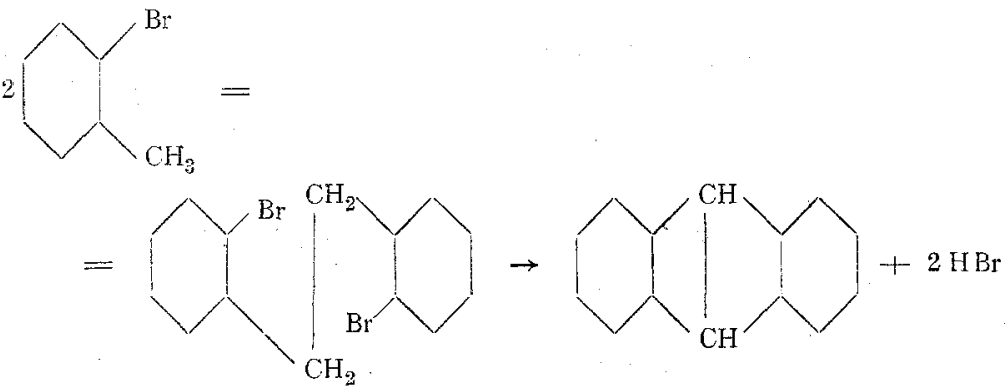

leicht verständlich ist.

I Wallach, Ann., 235, 271 (1886). 
Vermutlich wird sich diese äußerst glatt verlaufende Synthese auch für die Darstellung substituierter Anthracene verwenden lassen, was wir untersuchen wollen.

Der geringe flüssige Anteil des Reaktionsproduktes lieferte bei der Oxydation mit Permanganat etwas Orthobrombenzoesäure und Anthrachinon, enthält also neben noch in Lösung verbliebenem Anthracen wahrscheinlich oo.Dibromstilben und o o-Dibromdiphenyl.

\section{Parachlortoluol.}

Der bei 220 bis $260^{\circ}$ übergehende Hauptanteil des Destillates wurde mit dem doppelten Volumen Petrolpentan vermischt und über Nacht im Weinhold'schen Gefäß in der Kohlensäure-Acetonkältemischung stehen gelassen. Es schieden sich dicke Krystallnadeln in großer Menge ab. Wiederholtes Umkrystallisieren aus Pentan ließ den Schmelzpunkt auf 110 bis $111^{\circ}$ steigen; die Mikrochlorbestimmung gab jetzt zwar den für ein Dichlordibenzyl berechneten Wert, ließ aber deutlich ersehen, daß die Substanz nicht ganz rein sei: ein Teil war nämlich merklich schwerer flüchtig. ${ }^{1}$

Die Substanz wurde deswegen nochmals fraktioniert destilliert und die leichter flüchtigen Anteile aus Hexan wiederholt umkrystallisiert.

Der Schmelzpunkt stieg noch um einen Grad, auf 111 bis $112^{\circ}$. Die Mikroanalyse erwies jetzt das Vorliegen einer reinen Substanz, die als Paradichlordibenzyl anzusprechen ist. Datta und Fernandes ${ }^{2}$ geben für diese Substanz den Schmelzpunkt $111^{\circ}, \mathrm{Kade}^{3} 112^{\circ}$ an.

Aus den Mutterlaugen wurde durch mühseliges Fraktionieren in kleinen Mengen ein zweiter Körper herauspräpariert, der in reinem Zustande wesentlich schwerer löslich ist und in größerer Menge erhalten wurde, als versucht wurde, durch länger andauerndes Erhitzen bei höherer Temperatur aus dem Dichlordibenzyl Dichloranthracen darzustellen. Die Substanz,

1 Die Mikroanalyse erwies sich hier, wie auch sonst oft, als ein Mittel, die Uneinheitlichkeit minimaler Substanzmengen zu erkennen.

, 2 Am. soc., 38, 1809 (1916).

3 J. pr. (2), 19, 462 (1879). 
wiederholt aus Hexan und Alkohol umkrystallisiert, schmilzt konstant bei 168 bis $171^{\circ}$. Sie bildet feine, silberglänzende Nadeln, die sich auf dem Filter zu einem dichten Filz zusammenlagern. Die Analyse und der Vergleich mit den für $p$-Dichlorstilben angegebenen Eigenschaften zeigten, daß diese Substanz vorliegt.

Durch den Eintritt des Halogens in das Molekül wird also bewirkt, daß die Äthanwasserstoffe früher abgespalten werden als die für den Ringschluß in Betracht kommenden Wasserstoffatome des Kernes. Daß aus den Stilbenen keine Anthracenbildung mehr erfolgt, haben wir schon in der ersten Mitteilung ausgeführt.

Das stibenhaltige Dichlordibenzyl ergab für $5 \cdot 04 \mathrm{mg}$ Substanz $5 \cdot 787 \mathrm{mg}$ Chlorsilber.

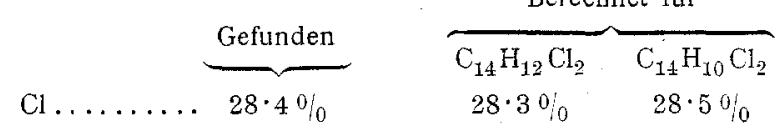

Das reine Dichlordibenzyl lieferte bei der Elementaranalyse aus $5.512 \mathrm{mg}$ $13 \cdot 528 \mathrm{mg} \mathrm{CO}_{2}$ und $2 \cdot 38 \mathrm{mg}$. Wasser.

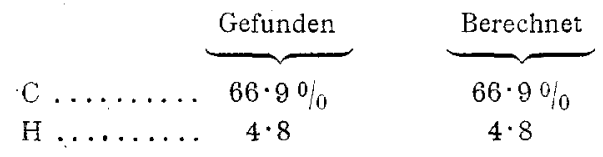

Das relne Dichlorstilben ergab ais $5 \cdot 648 \mathrm{mg} 13 \cdot 898 \mathrm{mg} \mathrm{CO}_{2}$ und $2 \cdot 19 \mathrm{mg}$ Wasser.

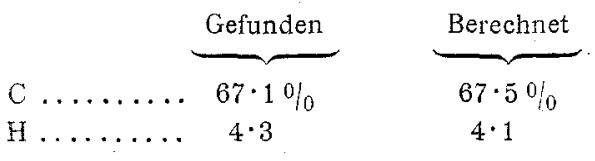

\section{p-Fluortoluol.}

Nach dem Abdestillieren des unveränderten Fluortoluols wurde eine Hauptfraktion von 270 bis $285^{\circ}$ und ein kleiner Nachlauf bis gegen $320^{\circ}$ aufgefangen. Beide Fraktionen erstarrten beim Abkühlen krystallinisch, die erstere fast vollständig. Nach dem Abpressen wurde aus Alkohol umkrystallisiert, bis der Schmelzpunkt bei $106^{\circ}$ konstant blieb. 
Das Produkt aus der Hauptfraktion war sofort farblos, das aus dem höher siedenden Anteil gelblich. Es wurde deshalb vermutet, daß es anthracenhaltig sei, doch erwies sich diese Annahme als irrig. Auch die gelbliche Substanz ließ sich unschwer reinigen. Sie ist mit dem Hauptprodukt identisch.

Die Reaktion verläuft hier rasch und bei nicht sehr hoher Temperatur. Wir mußten daher erwarten, daß die Substanz das $p p$-Difluordibenzyl sei. Präparate von drei verschiedenen Darstellungen - es sei erwähnt, daß es uns nicht immer gelungen ist, den Schmelzpunkt bis auf $106^{\circ}$ hinaufzutreiben ergaben aber genau auf das zugehörige Stilben stimmende Analysenwerte.

Präparat I. Schmelzpunkt 103 bis $104^{\circ} .1$

Präparat II. Schmelzpunkt $106^{\circ}, 4.969 \mathrm{mg}$ gaben $14.086 \mathrm{mg}^{-} \mathrm{CO}_{2}$ und $2 \cdot 01 \mathrm{mg}$ Wasser.

Präparat III. Schmelzpunkt 105 bis $106^{\circ}$. $5.634 \mathrm{mg}$ gaben $15.99 \mathrm{mg} \mathrm{CO}_{2}$ und $2 \cdot 48 m g$ Wasser.

\begin{tabular}{|c|c|c|c|c|c|}
\hline & \multicolumn{2}{|c|}{ Berechnet für } & \multicolumn{3}{|c|}{ Gefunden } \\
\hline & $\mathrm{C}_{14} \mathrm{H}_{12} \mathrm{~F}_{2}$ & $\mathrm{C}_{14} \mathrm{H}_{10} \mathrm{~F}_{2}$ & I & II & IH \\
\hline & $77.0 \%$ & $77 \cdot 7 \%$ & $77 \cdot 50: 0$ & $77 \cdot 3 \%$ & $77 \cdot 4 \%$ \\
\hline . . & $5 \cdot 5$ & $4 \cdot 7$ & $4 \cdot 6$ & $4 \cdot 5$ & $4 \cdot 9$ \\
\hline
\end{tabular}

Das Difluordibenzyl ist also offenbar sehr wenig hitzebeständig. Wahrscheinlich ist es in der kleinen Menge des Öls enthalten, das als Nebenprodukt erhalten wird. Wir haben versucht, das in diesem Öl als zweiter Bestandteil noch vorhandene gelöste Difluorstilben durch Natriumamalgam zu reduzieren und dadurch das jedenfalls durch diesen zweiten Körper am Festwerden gehinderte Äthanderivat zum Krystallisieren zu bringen, aber das Stilben erwies sich sowohl in diesem Gemisch als auch in reiner Form als nicht reduzierbar, was übrigens, nach den bekannten Erfahrungen an anderen Stilbenen, zu erwarten gewesen war.

1 Die Aufzeichnung über die Wägungen für diese Analyse ist uns zur Zeit nicht zugänglich. 
Daß in dem Öl keine anderen Bestandteile vorliegen, ist außer durch den Siedepunkt durch das Resultat der Oxydation mit Permanganat bewiesen, das nur Parafluorbenzoesäure vom Schmelzpunkt $182^{\circ}$ ergab, die nach dem Verfahren von Hans Meyer und $\mathrm{Hub}^{1}$ durch Überführen in den bei 4 bis $5^{\circ}$ schmelzenden Methylester identifiziert wurde.

Nicht nur gegen Wasserstoff, sondern auch gegen Brom ist das Difluorstilben recht resistent. Läßt man aber die Lösung des Stilbens in Eisessig mehrere Wochen lang mit Brom-Eisessig stehen, so krystallisieren schöne, kompakte Nadęln aus, die in Alkohol sehr schwer löslich sind. Aus Eisessig und aus viel Alkohol umkrystallisiert, schmilzt die Substanz konstant bei 141 bis $142^{\circ}$.

Sie enthält viel Brom, wie qualitativ festgestellt wurde, und ist als das $p p$-Difluor-1.2-dibromdibenzyl

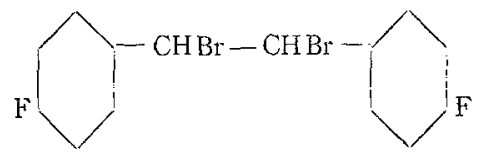

anzusehen. Die Mikrobrombestimmung nach dem Pregl'schen Verfahren auszuführen gelang nicht, da infolge von Fluorabspaltung Fluorsilicium, respektive Kieselfluorwasserstoffsäure entsteht, was zur Verunreinigung des Bromsilbers mit Kieselsäure und Kieselfluorsilber führt, die sich nicht vollständig entfernen ließen. Die Resultate wurden deshalb immer zu hoch gefunden. ${ }^{2}$

Sowohl der Schmelzpunkt des Difluordibromdibenzols als auch der des Difluorstilbens ist bemerkenswert niedrig, da ja 1.2-Dibromdibenzyl bei $257^{\circ}$ und Stilben bei $124^{\circ}$ schmilzt. Wir finden aber auch sonst die Erscheinung, daß der Eintritt von Fluor in das Molekül den Schmelzpunkt und den Siedepunkt ${ }^{3}$ erniedrigen kann.

1 Monatsh., 31, 935 (1910).

2 Herr Dr. Eckert ist damit beschäftigt, eine Mikrofluoranalyse und eine Methode zur Halogenbestimmung in fluorhaltigen Substanzen auszuarbeiten.

3 Hans Meyer und $\mathrm{Hub}$, Monatsh., 31, 935- (1910). 


\section{Metafluortoluo1.}

Die nach dem Abtrennen des Vorlaufes erhaltene, bis gegen $320^{\circ}$ siedende Flüssigkeit wurde beim Abkühlen bis auf $-80^{\circ}$ nur glasig. Dagegen gelang die Abscheidung reichlicher Mengen von Krystallen, als das Reaktionsprodukt in Pentan aufgenommen und unter oftmaligem kräftigen Reiben in dem Weinhold'schen Gefäß längere Zeit im KohlendioxydÄtherkältegemisch belassen wurde.

Die Krystalle wurden auf gekühlte Tonplatten gebracht. Nach dem Umkrystallisieren aus wenig Alkohol wurden prächtige, glänzende Krystalle (breite Lamellen) erhalten, deren Schmelzpunkt $38^{\circ}$ sich beim wiederholten Umkrystallisieren nicht mehr änderte.

Die Analyse ergab das Vorliegen von $m$-Difluordibenzyl.

$3 \cdot 727 \mathrm{mg}$ lieferten $10 \cdot 545 \mathrm{mg} \mathrm{CO}_{2}$ und $1 \cdot 81 \mathrm{mg}$ Wasser.

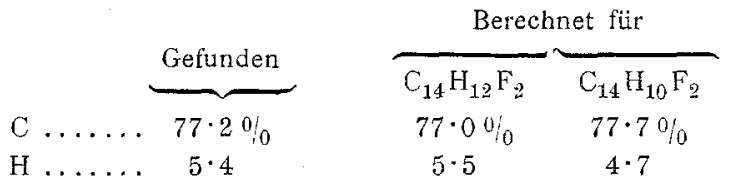

Während also beim Parafluortoluol als Hauptprodukt das entsprechende Stilben erhalten wurde, liefert das Metafluortoluol im wesentlichen das Äthanderivat: die lockernde Wirkung, die das Fluor aus der Parastellung ausübt, ist aus der Metastellung nicht bemerkbar.

\section{Parajodtoluo1.}

Aus dem Vorlauf wurde nach dem Behandeln mit Bisulfit, Trocknen und nochmaligem Fraktionieren etwas Toluol abgeschieden und durch Überführen in Dinitrotoluol identifiziert.

Die Verarbeitung des eigentlichen Reaktionsproduktes wurde hier in etwas abweichender Weise vorgenommen.

Zunächst wurde mit Bisulfitlösung am Rückflußkühler gekocht, die nach dem Erkalten noch dunkle, erstarrte Masse gepulvert und wiederholt mit Toluol ausgekocht. Bis auf wenig 
einer amorphen, dunklen Substanz ging alles in Lösung. Die Toluollösung wurde siedend filtriert, mit Tierkohle gekocht und nach nochmaligem Filtrieren konzentriert. Nach dem Erkalten wurde eine reichliche Ausscheidung gelblicher Krystalle abgesaugt.

Die Mutterlauge wurde destilliert. Nach dem Abtrennen des Jodtoluols und Toluols wurde ein zwischen 270 bis $330^{\circ}$ übergehendes Öl erhalten, aus dessen ersten Anteilen Dibenzyl in reiner und Stilben in nahezu reiner Form herauspräpariert werden konnten. Der höhersiedende Anteil wurde mit Permanganat oxydiert, aber außer ein wenig (nicht ganz reiner) Benzoesäure wurde nichts erhalten. Die Hauptmenge der Substanz war vollkommen zerstört.

Die Krystalle aus der Toluollösung wurden nun aus einem Gemisch von Alkohol (in dem allein die Substanz auch in der Hitze nur sehr schwer löslich ist) und Toluol unter Tierkohlezusatz umkrystallisiert. Es wurden so hübsche, farblose Blättchen erhalten, deren Schmelzpunkt bei 257 bis $259^{\circ}$ lag. Die Substanz sublimiert sehr schön. Der Schmelzpunkt wird durch das Sublimieren nicht verändert.

I. Mikroelementaranalyse. 1

II. $6 \cdot 737 \mathrm{ng}$ gaben $7 \cdot 285 \mathrm{mg}$ Jodsilber.

\begin{tabular}{|c|c|c|c|c|}
\hline & $\mathrm{Ge}$ & den & Berec & net für \\
\hline & I & II & $\mathrm{C}_{14} \mathrm{H}_{12} \mathrm{~J}_{2}$ & $\mathrm{C}_{14} \mathrm{H}_{10} \mathrm{~J}_{2}$ \\
\hline $\mathrm{C} \ldots \ldots$ & $38 \cdot 8010$ & - & $38 \cdot 6 \%$ & $38 \cdot 9 \%$ \\
\hline$H \ldots \ldots \ldots$ & $2 \cdot 7$ & - & $3 \cdot 0$ & $2 \cdot 3$ \\
\hline$J \ldots \ldots \ldots$ & - & $58 \cdot 5 \%$ & $58 \cdot 4$ & $58 \cdot 7$ \\
\hline
\end{tabular}

Die analytischen Daten zeigen, daß ein Stilben vorliegt. Die Substanz ist, dem hohen Schmelzpunkt entsprechend, als Paradijodstilben

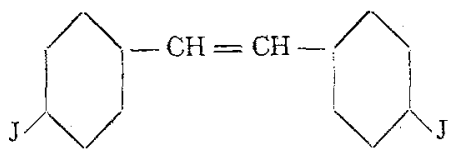

anzusehen.

1 Siehe Anm. 1 auf Seite $1 \tilde{3} 3$. 


\section{Benzylchlorid.}

Benzylchlorid ist schon von $\mathrm{L}_{0} \mathrm{~b}^{1}$ der Überhitzungsreaktion unterworfen worden. Er fand ausschließlich Stilber, vermutet aber, daß bei höherer Temperatur auch Phenanthren entstehen würde. Wir beobachteten - auch bei hoher Temperatur und andauerndem Erhitzen - neben Stilben und dessen flüssigen oder harzigen Polymerisationsprodukten nur ein wenig Toluol und eine Spur Dibenzyl, aber durchaus kein Phenanthren, entsprechend unsern früher ${ }^{2}$ geäußerten Anschauungen.

I. Z. Elekir。 9, 906 (1903).

2 Monatsh. 37, 696 (1916). 